\title{
Soja transgênica BRS 243 RR: determinação de macronutrientes e das isoflavonas daidzeína e genisteína por Cromatografia Líquida de Alta Eficiência (CLAE)
}

\author{
Transgenic soybean BRS 243 RR: determination of macronutrients and isoflavones \\ daidzein and genistein by High Performance Liquid Chromatography (HPLC)
}

\author{
Marcela Roquim ALEZANDRO ${ }^{1}$, Sandra Aparecida de ALMEIDA², Patrícia Penido MAIA3, \\ Helenice Aparecida de CARVALHO $^{4}$, Luciana AZEVEDO ${ }^{2 *}$, Elisabeth Pizzamiglio VIEIRA ${ }^{3}$
}

\section{Resumo}

Este trabalho teve por objetivo determinar a composição centesimal e o conteúdo de Daidzeína (D) e Genisteína (G) da cultivar BRS 243 RR por CLAE. O preparo da amostra para cromatografia envolveu a remoção da gordura com hexano . Os analitos foram extraídos com etanol $70 \%$ acrescido de $0,1 \%$ de ácido acético. As condições cromatográficas otimizadas foram: coluna $\mathrm{C}_{18}$, fase móvel metanol: ácido acético $5 \%$ $(1: 1 \mathrm{v} / \mathrm{v})$, vazão $0,5 \mathrm{~mL} /$ minuto, temperatura da coluna $30^{\circ} \mathrm{C}$, volume de injeção $40 \mu \mathrm{L}$ e leitura em $254 \mathrm{~nm}$. Os parâmetros de validação avaliados foram: linearidade $y=11242 x-37433, r=0,9976$ (D) e y $=18510 x-66761, r=0,9980(G)$; coeficientes de variação dos estudos de precisão intradia $C V=5,3 \%(\mathrm{D}), \mathrm{CV}=6,7 \%(\mathrm{G})$ e interdias $C V=8,7 \%(\mathrm{D}), \mathrm{CV}=9,7 \%(\mathrm{G})$; limite de quantificação $10 \mu \mathrm{g}$.g ${ }^{-1}$; limite de detecção $5 \mu \mathrm{g} \cdot \mathrm{g}^{-1}$ e recuperação $95,7 \%$. Portanto, o método desenvolvido foi adequado para a determinação de daidzeína e genisteína em soja. Os níveis de carboidratos (31,4\%), proteínas (35,9\%), lipídios (20,9\%), umidade (6,9\%), cinzas (4,9\%), daidzeína $(44,1 \mu$ g.g-1 $)$ e genisteína $\left(37,4 \mu \mathrm{g} \cdot \mathrm{g}^{-1}\right)$ determinados na soja transgênica foram similares aos de outros estudos com soja convencional.

Palavras-chave: soja transgênica; isoflavonas; daidzeína; genisteína; composição centesimal; CLAE.

\begin{abstract}
The objective of this work was to evaluate the proximate composition, as well as Daidzein (D) and Genistein (G) contents by HPLC, of BRS 243 RR soybean. Sample preparation for the chromatographic analysis involved the use of hexane to remove lipids. Isoflavones were extracted with $70 \%$ ethanol containing $0.1 \%$ acetic acid. The optimized chromatographic conditions were: $\mathrm{C}_{18}$ column, mobile phase methanol:5\% acetic acid $(1: 1 \mathrm{v} / \mathrm{v})$, flow rate $0.5 \mathrm{~mL} /$ minute, column temperature $30^{\circ} \mathrm{C}$, $\mathrm{UV}$ absorbance at $254 \mathrm{~nm}$ and volume injected $40 \mu \mathrm{L}$. The validation parameters were: linearity of daidzein $(y=11242 \mathrm{x}-37433, \mathrm{r}=0.9976)$ and genistein $(\mathrm{y}=18510 \mathrm{x}-66761, \mathrm{r}=0.9980)$; variation coefficients obtained in intra-day precision assays $[\mathrm{CV}=5.3 \%(\mathrm{D}), \mathrm{CV}=6.7 \%(\mathrm{G})]$ and inter-day precision assays $[\mathrm{CV}=8.7 \%(\mathrm{D}), \mathrm{CV}=9.7 \%(\mathrm{G})]$; quantification limit $10 \mu \mathrm{g} . \mathrm{g}^{-1}$; detection limit $5 \mu \mathrm{g} . \mathrm{g}^{-1}$ and recovery $95.7 \%$. Therefore, the optimized method was appropriate for the determination of daidzein and genistein in soybean. Carbohydrate (31.4\%), protein (35.9\%), lipid (20.9\%), moisture (6.9\%), ash (4.9\%), daidzein (44.1 $\mu$ g. $\left.\mathrm{g}^{-1}\right)$ and genistein $\left(37.4 \mu \mathrm{g} . \mathrm{g}^{-1}\right)$ contents determined in transgenic soybean were similar to those of conventional soybean determined in other studies.

Keywords: transgenic soybean; isoflavones; daidzein; genistein; centesimal composition; HPLC.
\end{abstract}

\section{Introdução}

No Brasil, o alimento geneticamente modificado de maior produção é a soja, sendo que a oferta de suas sementes no mercado nacional é da ordem de $60 \%$. A versatilidade dos grãos de soja dá origem a produtos e subprodutos muito utilizados na agroindústria, na indústria química e na de alimentos, o que determina o seu grande consumo por parte da população (EMBRAPA, 2005).

A despeito dos questionamentos dos efeitos adversos do consumo de soja transgênica, deve-se considerar também que essa leguminosa, na sua forma convencional, tem sido amplamente apontada como agente de prevenção ao câncer (CHEN et al., 2005; GUTIERREZ et al., 2005; INAGAKI et al., 2005). Importantes efeitos biológicos têm sido atribuídos às isoflavonas, como atividade antioxidante (ESAKI et al., 1999; NAIM et al., 1976), antiaterogênica (HUI et al., 2001), hipocolesterolêmica (LUI et al., 2003), propriedades estrogênicas (PARK et al., 2001), além do potencial anticarcinogênico (COWARD et al., 1993) e inibição da atividade enzimática (ESTEVES; MONTEIRO,

Recebido para publicação em 15/1/2007

Aceito para publicação em 28/5/2008 (002212)

Bioquímica e Biologia Molecular de Alimentos, Laboratório de Química, Departamento de Alimentos e Nutrição Experimental, Faculdade de Ciências Farmacêuticas,

Universade de São Paulo - USP, Av. Professor Lineu Prestes, 580, Bloco 14, CEP 05508-900, São Paulo - SP, Brasil, E-mail: mroquim@usp.br

${ }^{2}$ Grupo de Pesquisa Alimentos e Nutrição, Laboratório de Nutrição Experimental, Departamento de Nutrição, Universidade Federal de Alfenas - UNIFAL,

Gabriel Monteiro da Silva, 700, Bairro Centro, CEP 37130-000, Alfenas - MG, Brasil, E-mail: lazevedo@unifal-mg.edu.br

3 Grupo de Pesquisa Análise Química de Toxicantes e Fármacos, Laboratório de Análises Toxicológicas, Departamento de Análises Clínicas,

Universidade Federal de Alfenas - Unifal, Gabriel Monteiro da Silva, 700, Bairro Centro, CEP 37130-000, Alfenas - MG, Brasil,

E-mail: beth@unifal-mg.edu.br, patpenido@unifal-mg.edu.br

${ }^{4}$ Grupo de pesquisa Alimentos e Nutrição, Laboratório de Bromatologia, Departamento de Farmácia, Universidade Federal de Alfenas - UNIFAL,

Gabriel Monteiro da Silva, 700, Bairro Centro, CEP 37130-000, Alfenas - MG, Brasil, E-mail: helenice@unifal-mg.edu.br

${ }^{*}$ A quem a correspondência deve ser enviada 
2001). Alguns estudos mais recentes relacionam a soja e as isoflavonas com um efeito favorável na massa óssea de mulheres pós-menopausa (MA et al., 2008).

As isoflavonas, pertencentes à classe dos fitoestrógenos, também conhecidas como isoflavonóides (ESTEVES; MONTEIRO, 2001), são o principal tipo de polifenol presente na soja (ESAKI et al., 1999; ROSTAGNO; PALMA; BARROSO, 2005) e em outras leguminosas (ESTEVES; MONTEIRO, 2001). Existem 12 tipos de isoflavonas, divididas em agliconas, gliconas e seus conjugados glicosídeos malonil e acetil. As isoflavonas livres sem a molécula de açúcar, denominadas agliconas, são consideradas as mais importantes presentes na soja. Entre elas, a daidzeína e a genisteína têm se destacado em virtude do seu maior potencial protetor à saúde humana. Além disso, apresentam-se em quantidades mais elevadas na soja dos que as outras isoflavonas (ESTEVES; MONTEIRO, 2001).

A avaliação das isoflavonas como componentes dietéticos com potencial efeito benéfico à saúde humana depende da quantificação desses compostos na soja e seus derivados. A cromatografia líquida de alta eficiência (CLAE) tem sido reconhecida como o método mais eficaz para a determinação das isoflavonas (COWARD et al., 1993; HUI et al., 2001; SONG, 1998).

De acordo com o Princípio da Equivalência Substancial, o alimento geneticamente modificado é seguro caso não possua diferença aparente entre ele e o alimento convencional. Sabe-se que a aplicação de glifosato, um herbicida muito utilizado no plantio de soja, altera significativamente sua composição química, por exemplo, o conteúdo de macronutrientes e o nível de compostos fenólicos, tais como isoflavonas (MILLSTONE; BRUNNER; MAYER, 2006). Sendo a aplicação de glifosato na soja transgênica superior à empregada na soja convencional, métodos que avaliem com precisão os níveis de isoflavonas, bem como a composição centesimal da soja submetida à ação desses herbicidas, são de grande relevância para detectar as possíveis variações desses componentes em relação à soja convencional.

Portanto, o objetivo deste trabalho foi determinar os níveis de daidzeína e genisteína, proteína, lipídios, umidade e cinzas de uma cultivar de soja geneticamente modificada.

\section{Material e métodos}

\subsection{Material}

A soja utilizada nas análises foi da cultivar BRS 243 RR, ciclo precoce, cor da flor branca, pubescência marrom, cor do hilo marrom, brilho do tegumento fosco, tipo de crescimento determinado, fornecida pela Embrapa Soja. Os padrões daidzeína e genisteína foram obtidos da Sigma Chemical Co. (St. Louis, Mo., USA). Os reagentes e solventes usados foram de grau analítico e/ou para cromatografia líquida.

\subsection{Métodos}

\section{Preparo da amostra}

Os grãos de soja foram triturados em moinho Fritsch Pulverisette 14 à velocidade de 20000 rpm, obtendo-se uma farinha com partículas de $0,5 \mathrm{~mm}$ de diâmetro, a qual foi utilizada nas análises de composição centesimal.

Para a análise cromatográfica, a farinha de soja foi desengordurada segundo a metodologia descrita por Fujita (2003) (Figura 1). Utilizou-se o hexano, na proporção de $1 \mathrm{~mL}$ : 0,5 g de farinha de soja, com agitação em ultra-som por 30 minutos, à temperatura ambiente. Posteriormente, a amostra foi filtrada em funil de Büchner e o excesso de solvente presente no resíduo foi evaporado à temperatura ambiente por 12 horas. A amostra seca foi dividida em alíquotas de $100 \mathrm{mg}$ e armazenada $\mathrm{a}-18^{\circ} \mathrm{C}$, ao abrigo da luz.

\section{Análise da composição centesimal}

As análises da composição centesimal foram realizadas em triplicata, de acordo com a metodologia preconizada pela Association of Official Analytical Chemists (1997). Assim, o teor de umidade foi determinado com utilização de estufa a $105^{\circ} \mathrm{C}$; as cinzas em mufla a $550{ }^{\circ} \mathrm{C}$; os lipídios pelo método de Soxhlet, empregando-se éter de petróleo, e as proteínas pelo método de micro-Kjeldahl, utilizando-se o fator de conversão de 6,25. A fração nifext (carboidratos) foi obtida pelo cálculo da diferença percentual entre os valores obtidos na determinação de umidade, cinzas, lipídios e proteína.

\section{Análise cromatográfica}

\section{Extração das isoflavonas}

O procedimento de extração das isoflavonas de soja foi realizado de acordo com metodologia proposta por Carrão-Panizzi et al. (2002).

Foram utilizados $100 \mathrm{mg}$ de farinha desengordurada de soja e $4 \mathrm{~mL}$ de solução extratora (concentração de $0,025 \mathrm{~g} \cdot \mathrm{mL}^{-1}$ ) composta por etanol a $70 \%$ com $0,1 \%$ de ácido acético. Durante o período de uma hora, a amostra foi submetida à agitação a cada

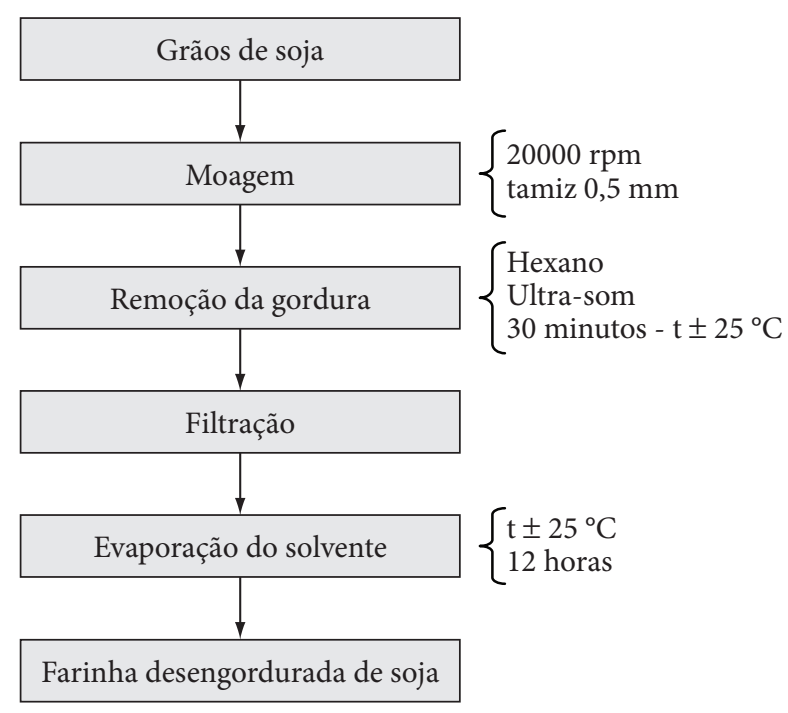

Figura 1. Fluxograma do preparo da farinha desengordurada de soja para análise cromatográfica. 
15 minutos, sendo cada agitação realizada em 30 segundos, em agitador de tubos do tipo vortex. Em seguida, os tubos contendo a amostra e a solução extratora foram expostos à sonicação em ultra-som durante 30 minutos, à temperatura ambiente.

Posteriormente, foram pipetados $1,5 \mathrm{~mL}$ do sobrenadante, os quais foram centrifugados a $1700 \mathrm{~g}$, por 15 minutos. $\mathrm{O}$ sobrenadante coletado foi filtrado em microfiltro de $0,45 \mu \mathrm{m}$, obtendo-se o extrato, o qual foi armazenado ao abrigo da luz, $\mathrm{a}-18^{\circ} \mathrm{C}$.

O fluxograma do processo de extração utilizado para determinação das isoflavonas da soja está disponível na Figura 2.

\section{Condições cromatográficas}

Como condições cromatográficas, foi utilizado o modo de eluição isocrático, com fase móvel composta por metanol: ácido acético $5 \%(1: 1 \mathrm{v} / \mathrm{v})$, vazão de $0,5 \mathrm{~mL} \cdot \mathrm{min}^{-1}$, temperatura do forno de $30^{\circ} \mathrm{C}$, leitura dos analitos em comprimento de onda de $254 \mathrm{~nm}$, não sendo utilizado padrão interno nas análises. Uma alíquota de $40 \mu \mathrm{L}$ foi injetada em cromatógrafo líquido Shimadzu série LC-10 ATVP com injetor automático e equipado com detector de UV e coluna cromatográfica LiChrospher 60 RP-Select B Merck ( 250 x 4 mm, $5 \mu \mathrm{m})$ e coluna de guarda $\mathrm{LiChCART}^{\circledast}$ 4-4.

\section{Parâmetros de validação}

Foram avaliados, em triplicata, os seguintes critérios de validação, linearidade, precisão intra e interdias, limite de

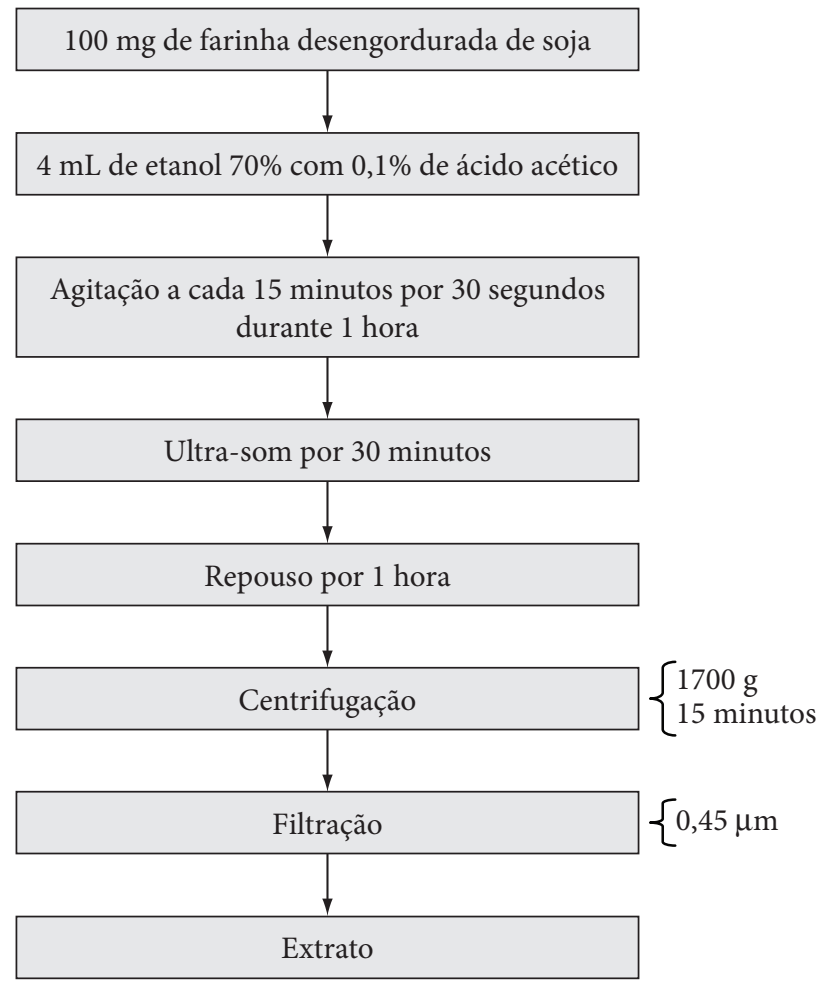

Figura 2. Fluxograma do procedimento de extração das isoflavonas da soja. detecção, limite de quantificação, recuperação e estabilidade, conforme a Agência Nacional de Vigilância Sanitária - ANVISA (2003). A linearidade foi obtida pela adição de daidzeína e genisteína ao extrato, obtendo-se as concentrações finais de: 10, 20, 40, 60, 80 e 100 ng. $\mu \mathrm{L}^{-1}$. O Limite de Detecção (LD) e o Limite de Quantificação (LQ) foram determinados utilizando-se soluções dos padrões daidzeína e genisteína em concentrações decrescentes.

A precisão interdias foi realizada com a injeção de $40 \mu \mathrm{L}$ do extrato adicionado de $20 \mathrm{ng}$ de genisteína e $20 \mathrm{ng}$ de daidzeína. Para a fortificação da amostra, utilizou-se uma solução de 1,0 ng. $\mu \mathrm{L}^{-1}$ de cada um dos padrões. Foram feitas análises durante cinco dias consecutivos.

O ensaio de precisão intradia foi realizado enriquecendose os extratos com soluções de padrão daidzeína e genisteína (1,0 ng. $\left.\mu \mathrm{L}^{-1}\right)$ nas concentrações de 10 a $100 \mathrm{ng} . \mu \mathrm{L}^{-1}$ e avaliado através dos coeficientes de variação obtidos.

A recuperação foi determinada comparando-se os resultados analíticos de amostras extraídas a partir de seis concentrações contemplando a faixa de linearidade $(10,20,40,60,80$ e 100 ng. $\left.\mu \mathrm{L}^{-1}\right)$, com os resultados obtidos com soluções padrão não extraídas, que representam 100\% de recuperação.

Para avaliar a estabilidade da amostra, foram preparados extratos puros (sem adição de padrão) com um intervalo de 47 dias. Para o preparo dos extratos, utilizou-se a mesma farinha de soja desengordurada, a qual foi mantida a $-18{ }^{\circ} \mathrm{C}$ ao abrigo da luz.

\section{Identificação e quantificação das isoflavonas}

A identificação foi feita pela comparação dos tempos de retenção dos padrões com os das amostras.

A quantificação foi realizada pela comparação entre a área absoluta obtida com a injeção da amostra e a área absoluta obtida com a injeção de uma quantidade conhecida de padrão.

\section{Resultados e discussão}

\subsection{Preparo da amostra}

Durante o preparo da farinha de soja utilizada na análise da composição centesimal, não houve emprego de aquecimento ou contato com solventes. Dessa forma, o conteúdo dos grupos homogêneos analisados na farinha reproduz com fidelidade os teores encontrados na soja íntegra.

Sabendo-se que a soja é um alimento com elevado conteúdo lipídico (7,1-21,9\% - peso fresco), o preparo da amostra para análise cromatográfica teve como finalidade principal a remoção de parte da gordura, alcançando-se a redução de 49,8\% do lipídio, diminuindo assim o nível de possíveis interferentes.

Acrescenta-se ainda, que o processo de obtenção da farinha de soja desengordurada não remove as isoflavonas ou suas formas glicosiladas da farinha integral, utilizando-se hexano como agente extrator (ELDRIDGE; KWOLEK, 1983; WANG; MURPHY, 1996). 


\subsection{Análise da composição centesimal}

A análise da composição centesimal contemplou a determinação dos teores de umidade, cinzas, lipídios e proteínas da farinha de soja.

Os componentes determinados para a farinha de soja transgênica foram comparados a dados referentes à soja convencional, estes disponíveis na literatura, conforme Tabela 1.

O intervalo dos teores da composição centesimal da soja convencional foi determinado utilizando-se o menor e o maior valor encontrado em tabelas de composição química de alimentos (FRANCO, 1999; IBGE, 1999; NEPA, 2004; PHILIPPI, 2001). Dessa forma, os teores dos componentes determinados para a soja transgênica analisada encontram-se dentro dos intervalos especificados. Segundo Park et al. (2002) e Lui et al. (2003), esses valores podem ser alterados em função do local de plantio, condições climáticas, fatores genéticos ou ainda, do método analítico utilizado para determinação desses componentes.

\subsection{Análise cromatográfica}

\section{Otimização das condições de extração das isoflavonas}

Foram testadas duas concentrações diferentes de amostra/solução extratora $\left(0,1\right.$ e 0,025 g.mL $\left.\mathrm{mL}^{-1}\right)$ e duas soluções extratoras, sendo uma composta por metanol $80 \%$ (LUI et al., 2003) e a outra por etanol $70 \%$ com $0,1 \%$ de ácido acético (AAc) (ANVISA, 2003). Os melhores resultados foram obtidos com a utilização de $100 \mathrm{mg}$ de farinha desengordurada de soja e $4 \mathrm{~mL}$ de solução extratora, na concentração de 0,025 g.mL $\mathrm{mL}^{-1}$ (Tabela 2). A solução extratora composta por etanol $70 \% \mathrm{com}$ $0,1 \%$ de AAc possibilitou maior recuperação das isoflavonas da soja e melhor definição dos picos no cromatograma, além de oferecer menor toxicidade tanto para o analista quanto para o meio ambiente. Os mesmos resultados foram obtidos nos estudos de Carrão-Panizzi et al. (1999; 2002).

Tabela 1. Comparação entre a composição centesimal da farinha de soja transgênica BRS 243 RR desengordurada e da soja convencional.

\begin{tabular}{lcc}
\hline Componente & $\begin{array}{c}\text { Soja BRS 243 RR } \\
(\%)\end{array}$ & $\begin{array}{c}\text { Soja convencional } \\
(\%)\end{array}$ \\
\hline Umidade & 6,9 & $5,6-10,2$ \\
Lipídios & 20,9 & $7,1-21,9$ \\
Proteínas & 35,9 & $14,0-36,5$ \\
Cinzas & 4,9 & $3,8-5,0$ \\
Carboidratos & 31,4 & $30,0-32,0$ \\
\hline${ }^{*}$ Fonte: Franco (1999); IBGE (1999); NEPA (1976); e Philippi (2002).
\end{tabular}

Tabela 2. Áreas absolutas das isoflavonas daidzeína e genisteína obtidas a partir da extração utilizando-se as concentrações de 0,1 e 0,025 g.mL $\mathrm{mL}^{-1}$, etanol $70 \%$ com $0,1 \%$ de ácido acético (AAc) como solução extratora, com ou sem o acréscimo de 1 hora de repouso.

\begin{tabular}{lcc}
\hline \multicolumn{1}{c}{$\begin{array}{c}\text { Concentração } \\
\left(\mathrm{g} . \mathrm{mL}^{-1}\right)\end{array}$} & Área da daidzeína & Área da genisteína \\
\hline 0,1 (sem repouso) & 236760 & 235588 \\
0,025 (sem repouso) & 170529 & 205745 \\
0,025 (com repouso) & 384863 & 986283 \\
\hline
\end{tabular}

Foi otimizado o tempo da extração com e sem o acréscimo de 1 hora de repouso posterior à agitação, considerando seu efeito sobre a área das isoflavonas. Foi possível avaliar que a utilização de 1 hora de repouso, totalizando 2 horas e 30 minutos de extração, permitiu obter melhor recuperação da daidzeína e da genisteína (Tabela 2).

As condições otimizadas para extração das isoflavonas não foram as mesmas utilizadas por Fujita (2003), Lui et al. (2003) e Park et al. (2001). Porém, os resultados obtidos são semelhantes ao método empregado no estudo realizado por Carrão-Panizzi et al. (2002).

O fluxograma do procedimento de otimização das condições de extração das isoflavonas da soja está apresentado na Figura 3.

\section{Otimização das condições cromatográficas}

A partir do estudo sobre o modo de eluição a ser empregado, três gradientes de concentração foram testados (AGUIAR, 2004; LUI et al., 2003). Nos três gradientes testados, a fase móvel era composta por AAc 5\% (solvente A) e metanol puro (solvente B). Em todos eles, observou-se um grande tempo de corrida (120 minutos), o que dificultou a análise de um maior número de amostras por dia. Dessa forma, optou-se pela utilização do modo de eluição isocrático, com um tempo total de corrida de 40 minutos, o que possibilita a análise de um maior número de amostras por dia.

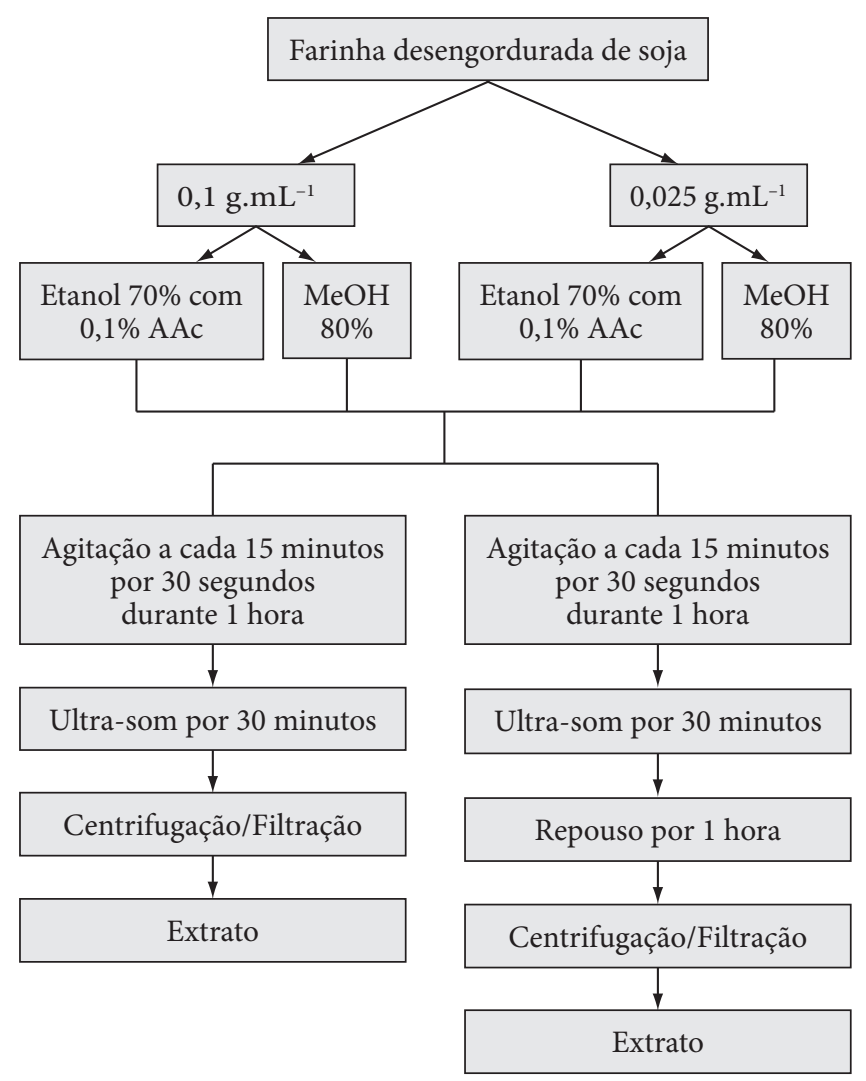

Figura 3. Fluxograma da otimização das condições de extração das isoflavonas. 
Com relação à fase móvel a ser utilizada, testaram-se duas composições distintas, uma composta por metanol: AAc 5\% $(1: 1 \mathrm{v} / \mathrm{v})$, sugerida por Park et al. (2002), e outra por AAc 5\%: metanol/acetonitrila (100/85) (1:1 v/v). Com a utilização desta última fase móvel, não foi possível a identificação dos picos referentes às isoflavonas daidzeína e genisteína. Isso resultou na utilização da fase móvel composta por metanol: AAc 5\% $(1: 1 \mathrm{v} / \mathrm{v})$, a qual permitiu uma adequada identificação dos picos referentes a essas mesmas isoflavonas.

Foram testadas duas vazões diferentes, uma de $0,5 \mathrm{~mL} /$ minuto (FUJTTA, 2003) e outra de $1,0 \mathrm{~mL} /$ minuto (CARRÃO-PANIZZI et al., 1999). A utilização de $1,0 \mathrm{~mL} /$ minuto não permitiu a identificação das isoflavonas no tempo de corrida determinado, optando-se pela vazão de $0,5 \mathrm{~mL} /$ minuto, a qual permitiu uma satisfatória localização dos picos de daidzeína e genisteína, além de utilizar uma menor quantidade de solvente e, conseqüentemente, menor impacto para o meio ambiente.

As temperaturas da coluna testadas foram de 30 e $40{ }^{\circ} \mathrm{C}$, obtendo-se melhores resultados a $30^{\circ} \mathrm{C}$. Segundo Coward et al. (1993), a utilização de temperaturas elevadas pode causar alterações na composição das isoflavonas, com formação dos outros tipos de isoflavonas, o que poderia alterar a determinação de daidzeína e genisteína.

Os comprimentos de onda testados foram de 254 e $262 \mathrm{~nm}$, e os melhores resultados foram obtidos com a utilização de $254 \mathrm{~nm}$, o que está de acordo com Klejdus et al. (2005) e Park et al. (2002).

Foram testados três volumes de injeção, sendo eles de 20, 40 e $50 \mu \mathrm{L}$, a partir dos quais foram obtidos melhores resultados com $40 \mu \mathrm{L}$, o mesmo utilizado no estudo de Carrão-Panizzi et al. (2002).

Os padrões internos testados, fluoresceína e kaempherol, não puderam ser utilizados, pois se mostraram incompatíveis com as condições de análise, em virtude dos seus tempos de retenção, os quais estavam próximos aos tempos de retenção das isoflavonas daidzeína e genisteína, ou por aumentarem muito o tempo da análise ( $\operatorname{tr}=36,1$ minutos), o que não reproduz os resultados encontrados nos estudos de Barnes, Kirk e Coward (1994) (Tabela 3).

\section{Parâmetros de validação}

O método analítico utilizado mostrou-se linear no intervalo de 10 a $100 \mathrm{ng} \cdot \mu \mathrm{L}^{-1}$, tanto para daidzeína quanto para genisteína, como está evidenciado nas Figuras 4 e 5.

Foi considerado limite de detecção a menor concentração que se diferenciou do zero com confiança, ou seja, que forneceu uma relação sinal/ruído igual a três. Já o limite de quantificação

Tabela 3. Padrões internos testados e média do tempo de retenção.

\begin{tabular}{lc}
\hline \multicolumn{1}{c}{ Padrão interno } & Tempo de retenção (minutos) \\
\hline Fluoresceína Merck $^{\infty}$ & 25,4 \\
Fluoresceína Sigma $^{\bullet}$ & 26,4 \\
Fluoresceína Hartman Leddon Co. $^{\circledR}$ & 25,4 \\
Kaempherol Merck $^{\oplus}$ & 36,1 \\
\hline
\end{tabular}

é a menor quantidade do analito que se pode quantificar com precisão aceitável, isto é, com coeficiente de variação menor que $10 \%$ (ANVISA, 2003). Nesse sentido, o limite de deteç̧ão obtido para o método foi de $5 \mu \mathrm{g} \cdot \mathrm{g}^{-1}$ e o limite de quantificação de $10 \mu \mathrm{g} \cdot \mathrm{g}^{-1}$ de daidzeína/genisteína na soja.

A precisão intradia é a máxima diferença aceitável entre suas repetições (LEITE, 1996), o que para analitos em matriz complexa é indicada através de coeficiente de variação menor que 10\% (ANVISA, 2003). A Tabela 4 mostra os coeficientes de variação obtidos nos estudos de precisão intradia e interdias do método. Todos os valores encontram-se dentro do limite permitido $(\mathrm{CV}<10 \%)$.

O estudo de recuperação mede a eficiência do procedimento de extração de um método analítico dentro de um limite de variação. $\mathrm{O}$ ensaio realizado indicou que o método de extração proporciona a recuperação de $95,7 \%$ das isoflavonas da farinha desengordurada de soja. Segundo a ANVISA (2003), porcentagens de recuperação do analito próximas a 100\% são desejáveis, porém, admitem-se valores menores, desde que a recuperação seja precisa e exata, o que demonstra que o método analítico utilizado apresenta boa recuperação.

$\mathrm{O}$ teste de estabilidade permitiu avaliar que a farinha desengordurada de soja mantém-se estável por até 47 dias, quando armazenada a $-18^{\circ} \mathrm{C}$ ao abrigo da luz.

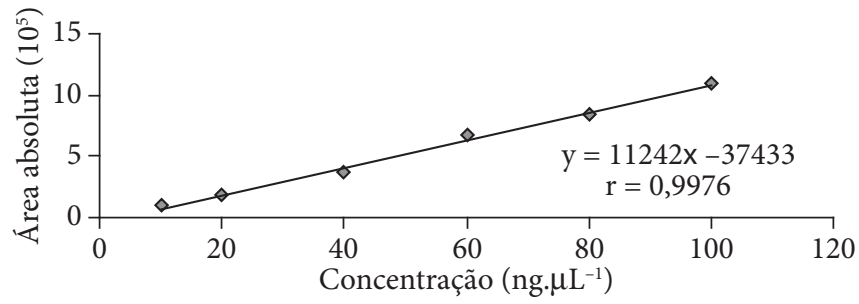

Figura 4. Curva analítica obtida para padrão daidzeína em fase móvel.

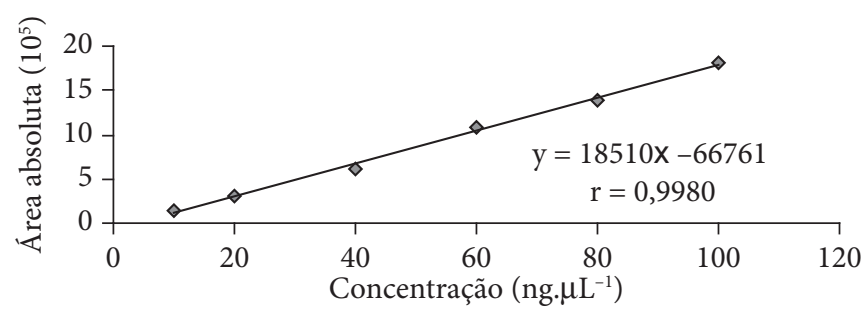

Figura 5. Curva analítica obtida para padrão genisteína em fase móvel.

Tabela 4. Coeficientes de variação obtidos nos ensaios de precisão intradias e interdias, em triplicata.

\begin{tabular}{ccc}
\hline \multirow{2}{*}{ Ensaio } & \multicolumn{2}{c}{ Coeficiente de variação (\%) } \\
\cline { 2 - 3 } & Daidzeína & Genisteína \\
\hline Precisão intradia & 5,3 & 6,7 \\
Precisão interdias & 8,7 & 9,7 \\
\hline
\end{tabular}




\section{Identificação e quantificação das isoflavonas}

As condições cromatográficas utilizadas para análise das isoflavonas permitiram a identificação dos picos referentes às isoflavonas daidzeína e genisteína, nos tempos 18,8 e 28,8 minutos, respectivamente, conforme está evidenciado na Figura 6.

A quantificação das isoflavonas permitiu avaliar que a soja analisada apresentava níveis de daidzeína e genisteína dentro do intervalo determinado pelo menor e pelo maior valor encontrado na literatura para soja convencional, como está descrito na Tabela 5.

É importante ressaltar, que o conteúdo das isoflavonas pode sofrer alterações de acordo com a cultivar, o local de plantio, solo, clima e a safra (HOECK et al., 2000).

\section{Conclusões}

Os componentes nutricionais que foram avaliados na determinação da composição centesimal apresentaram-se similares aos determinados por outros autores para soja convencional.

Os métodos de extração e de análise cromatográfica desenvolvidos foram adequados para a determinação das isoflavonas daidzeína e genisteína. Além disso, mostraram condições favoráveis à sua aplicação na determinação de daidzeína e genisteína em outras cultivares de soja, sejam elas convencional, transgênica ou orgânica.

Os teores de daidzeína e genisteína determinados na cultivar BRS 243 RR estão de acordo com os preconizados na literatura para soja convencional.

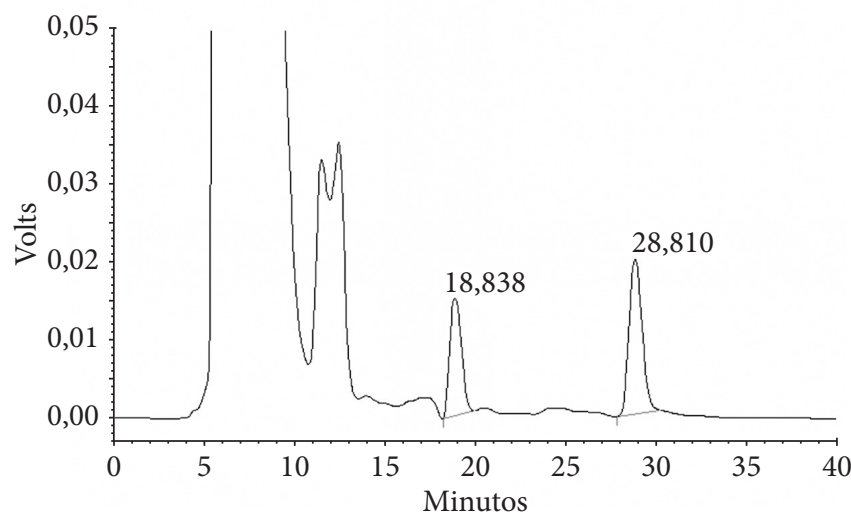

Figura 6. Cromatograma das isoflavonas daidzeína e genisteína sob as condições: coluna C18, fase móvel metanol:ácido acético 5\% (1:1 v/v), vazão $0,5 \mathrm{~mL} /$ minuto, temperatura do forno $30^{\circ} \mathrm{C}$, volume de injeção $40 \mu \mathrm{L}$, leitura a $254 \mathrm{~nm}$.

Tabela 5. Conteúdo de isoflavonas determinado na soja transgênica BRS 243 RR e os valores disponíveis na literatura para soja convencional.

\begin{tabular}{ccc}
\hline Isoflavonas & $\begin{array}{c}\text { Soja BRS 243 RR } \\
\left(\mu \mathrm{g} \cdot \mathrm{g}^{-1}\right)\end{array}$ & $\begin{array}{c}\text { Soja convencional } \\
\left(\mu \mathrm{g} \cdot \mathrm{g}^{-1}\right)\end{array}$ \\
\hline Daidzeína & 44,1 & $35,7-47,0$ \\
Genisteína & 37,4 & $32,0-60,4$ \\
\hline
\end{tabular}

*Fonte: Franke et al. (1999); Lui et al. (2003); e Park et al. (2002).
Dessa forma, pelo conceito de equivalência substancial, a cultivar de soja transgênica, em relação aos parâmetros analisados, é equivalente às variedades convencionais. Entretanto, é prudente a realização de testes biológicos, toxicológicos e imunológicos mais aprofundados e eficazes para avaliação da segurança quanto ao consumo de alimentos geneticamente modificados.

\section{Agradecimentos}

Os autores agradecem à Embrapa Soja pela disponibilização das cultivares utilizadas no experimento, ao programa de Iniciação Científica PROBIC/UNIFAL-MG e à FAPEMIG pelo apoio financeiro (processo CDS 1314/06).

\section{Referências bibliográficas}

AGUIAR, C. L. Transformações física e bioquímica de isoflavonas conjugadas de soja (Glycine Max L.) e o efeito na atividade biológica in vitro. Campinas, 2004. 285 f. Dissertação - (Doutorado em Ciências de Alimentos), Faculdade de Engenharia de Alimentos, Universidade Estadual de Campinas.

ANVISA - Agência Nacional de Vigilância Sanitária. Resolução RE $n^{\circ} 899$, de 29 de maio de 2003. Guia para validação de métodos analíticos e bioanalíticos. Diário Oficial da União, Brasília, 2003.

AOAC - Association of Official Analytical Chemistry. Official Methods of Methods of Analysis of de AOAC International: Supplement. 16 ed. Gaithersburg: AOAC, 1997. v. 2

BARNES, S.; KIRK, M.; COWARD, L. Isoflavones and their conjugates in soy foods: extraction conditions and analysis by HPLC-mass spectrometry. Journal Agricultural and Food Chemistry, v. 42, n. 11, p. 2466-2474, 1994.

CARRÃO-PANIZZI, M. C. et al. Effects of genetics and environment on isoflavone content of soybean from different regions of Brazil. Pesquisa Agropececuária Brasileira, v. 34, n. 10, p. 1787-1795, 1999.

CARRÃO-PANIZZI, M. C. et al. Extraction time for soybean isoflavone determination. Brazilian Archieves of Biology and Technology, v. 45, n. 4 , p. $515-518,2002$.

CHEN, Y. C. et al. Diet, vegetarian food and prostate carcinoma among men in Taiwan. British Journal of Cancer, v. 93, n. 9, p. 1057-1061, 2005.

COWARD, L. et al. Genistein, daidzein, and their $\beta$-glycoside conjugates: antitumor isoflavones in soybean foods from american and asian diets. Journal Agricultural and Food Chemistry, v. 41, n. 11, p. 1961-1967, 1993.

ELDRIDGE, A. C.; KWOLEK, W. F. Soybean isoflavones: effect of environment and variety on composition. Journal Agricultural and Food Chemistry, v. 42, n. 2, p. 394-396, 1983.

EMBRAPA - Empresa Brasileira de Pesquisa Agropecuária. Usos. Disponível em: http:<//www.cnpso.embrapa.br/indez.php?op_ page $=25$ \& cod_pai=29>. Acesso em: 08 out. 2005.

EMBRAPA - Empresa Brasileira de Pesquisa Agropecuária. Grãos e Fibras. Disponível em: http: $</ /$ www21.sede.embrapa.br/linhas_ de_acao/alimentos/grao_fibra/index_html/mostra_documento $>$. Acesso em: 09 out. 2005.

ESAKI, H. et al. Formation mechanism for potent antioxidative o-dihydroxyisoflavones in soybeans fermented with Aspergillus saitoi. Bioscience, Biotechnology and Biochemistry, v. 63, n. 5, p. 851-858, 1999. 
ESTEVES, E. A.; MONTEIRO, J. B. R. Efeitos benéficos das isoflavonas de soja em doenças crônicas. Revista de Nutrição, v. 14, n. 1, p. 43-52, 2001.

FRANCO, G. Tabela de composição química dos alimentos. 9 ed. São Paulo: Atheneu, 1999. 307p.

FRANKE, A. A. et al. Isoflavone levels in soy foods consumed by multiethinic populations in Singapore and Hawai. Journal Agricultural and Food Chemistry, v. 47, n. 3, p. 977-986, 1999.

FUJITA, A. Produção e caracterização de $\beta$-glicosidase vegetal e microbiana e sua aplicação para conversão de isoflavonas glicosiladas em isoflavonas agliconas. Campinas, 2003. $146 \mathrm{f}$. Dissertação - (Mestrado em Ciência de Alimentos), Faculdade de Engenharia de Alimentos, Universidade Estadual de Campinas.

GUTIERREZ, Z. et al. Soy isoflavone glycitein protects against beta amyloid-induced toxicity and oxidative stress in transgenic Caenorhabditis elegans. BMC-Neuroscience, v. 6, n. 54, p. 1471-2202, 2005.

HOECK, J. et al. Influence of genotype and environment contents of soybean. Crop Science, v. 40, p. 48-51, jan-fev. 2000.

HUI, E. et al. Genistein and daidzein/glycitein content in tofu. Journal Food Composition and Analysis, v. 14, n. 2 p. 199-206, 2001.

IBGE - Instituto Brasileiro de Geografia e Estatística. Tabela de composição de alimentos. 5 ed. Rio de Janeiro: IBGE, 1999. $137 \mathrm{p}$.

INAGAKI, K. et al. Suppression of urokinase expression and invasion by a soybean Kunitz trypsin inhibitor are mediated through inhibition of Src-dependent signaling pathways. Journal of Biological Chemistry, v. 280, n. 36, p. 31428-31437, 2005.

KLEJDUS, B. et al. Determination of isoflavones in soy bits by fast column high-performance liquid chromatography coupled with UV-visible diode-array detection. Journal of Chromatogragy A, v. 1084, n. 1-2, p. 71-79, 2005.

LEITE, P. R. Solventes Orgânicos. In: OGA, S. Fundamentos da Toxicologia. 1 ed. São Paulo: Atheneu Editora, 1996. p. 187-229.
LUI, M. C. Y. et al. Isoflavonas em isolados e concentrados protéicos de soja. Ciência e Tecnologia de Alimentos, v. 23, supl., p. 206-212, 2003.

MA, D. et al. Soy isoflavone intake increases bone mineral density in the spine of menopausal women: Meta-analysis of randomized controlled trials. Clinical Nutrition, v. 27, n. 1, p. 57-64, 2008.

MILLSTONE, E.; BRUNNER, E.; MAYER, S. Para além da "equivalência substancial". Nature, Brighton, v 401, p. 525-526, 07. out. 1999. Disponível em: http:<//www.aspta.org.br/publique/ media/equivsubst.pdf $>$. Acesso em: 06 mar. 2006.

NAIM, M. et al. Antioxidative and antihemolytic activities of soybean isoflavones. Journal Agricultural and Food Chemistry, v. 24, n. 6, p. 1174-1177, 1976.

NEPA - Núcleo de Estudos e Pesquisas em Alimentação. Tabela brasileira de composição de alimentos. Campinas: NEPA-UNICAMP, 2004. $42 \mathrm{p}$.

PARK, Y. K. et al. Biotransformações de isoflavonas de soja. Biotecnologia, Ciência \& Desenvolvimento, n. 20, p. 12-14, 2001.

PARK, Y. K. et al. Conversão de malonil- $\beta$-glicosil isoflavonas em isoflavonas glicosiladas presentes em alguns cultivares de soja brasileira. Ciência e Tecnologia de Alimentos, v. 22, n. 2, p. 130135, 2002.

PHILIPPI, S. T. Tabela de composição de alimentos: suporte para decisão nutricional. Brasília: ANVISA, FINATEC/NUT-UnB, 2001. 107p.

ROSTAGNO, M. A.; PALMA, M.; BARROSO, C. G. Solid-phase extraction of soy isoflavones. Journal of Chromatografy A, v. 1076, n 1-2, p. 110-117, 2005.

SONG, T. Soy isoflavone analysis: quality control and a new internal standard. American Journal of Clinical Nutrition, v. 68, supl., p. 1474S-1479S, 1998.

WANG, H. J.; MURPHY, P. A. Mass balance study of isoflavones during soybean processing. Journal Agricultural and Food Chemistry, v. 44, n. 8 , p. $2377-2383,1996$. 